\title{
Familial multiple sclerosis: MRI findings in clinically affected and unaffected siblings
}

\author{
P J Tienari, O Salonen, J Wikström, L Valanne, J Palo
}

\begin{abstract}
Subclinical demyelinating lesions may occur in the brains of asymptomatic individuals, and the first-degree relatives of multiple sclerosis (MS) patients are at particular risk. Clinical and MRI examinations were performed in nine sibships from families with two or more cases of MS. These included 14 patients with clinically definite MS, three patients with clinically probable $M S$, and 27 asymptomatic siblings. Systematic criteria were applied to MRI interpretations to increase their specificity for MS. Thirteen $(76 \%)$ of the 17 patients with MS showed lesions suggesting MS. Lesions were also found in six $(38 \%)$ of the 16 asymptomatic siblings under age 50 and in eight (73\%) of the 11 over age 50 . Judged by stringent criteria, the lesions of only three $(11 \%)$ of the 27 asymptomatic siblings were considered to be due to demyelination. The results demonstrate the occurrence of subclinical demyelination in asymptomatic siblings of MS patients and stress the importance of clinical follow up and MRI studies of the first-degree relatives when classifying them as healthy in family studies.
\end{abstract}

(F Neurol Neurosurg Psychiatry 1992;55:883-886)

Estimates of the familial incidence of multiple sclerosis (MS) vary from 3.6 to $20 \% .{ }^{1-6}$ In one high risk area of Finland a prevalence of $8 \%$ has been calculated for living siblings of the patients. $^{7}$ There are reports suggesting that subclinical disease may be present in asymptomatic individuals, especially if they have a family history of MS. Among monozygotic twins discordant for MS, typical MRI plaques and CSF oligoclonal bands can be found in unaffected twins. ${ }^{8-10}$ Further, oligoclonal bands and abnormal evoked potentials have been documented among clinically healthy siblings of MS patients. ${ }^{112}$ MS plaques have also been detected in asymptomatic individuals at necropsy. ${ }^{1314}$

The most sensitive method for showing CNS white matter lesions in MS is MRI. ${ }^{15} \mathrm{Up}$ to $95 \%$ of clinically definite cases, and $50-80 \%$ of clinically probable cases show these lesions. ${ }^{15}{ }^{18} \mathrm{MRI}$ is therefore today the most reliable technique to study the occurrence of subclinical MS. However, only one study of MRI findings among asymptomatic subjects (other than twins) in MS families has been published. ${ }^{19}$ The results support the concept that subclinical cases may be found in apparently healthy subjects but no detailed analysis of the specificity of the white matter lesions nor familial distribution of the findings were presented. To answer these questions and to increase diagnostic certainty for genetic studies we examined clinically and with MRI the siblings of nine families with two or more cases of MS. Six of the families are from a very high risk area in Finland (the district of Vaasa) where the epidemiology of the disease has been followed up since $1964 .^{20}$

\section{Subjects and methods}

Nine sibships from families witn at least two MS cases were chosen with a total of 48 individuals. All siblings took part in the study; $18(38 \%)$ of them had MS (nine females and nine males). Fifteen patients had clinically definite MS (CDMS) and three had clinically probable MS (CPMS) according to Poser's diagnostic criteria. ${ }^{21}$ All MS patients had remitting-relapsing disease, and all except one were examined during remission. Their mean age was 50 years (range 33-69 years). The mean duration of the disease was 21 years (11-38 years). Of the sibships six (I, II, III, IV, VIII and IX) come from a very high risk area ${ }^{20}$ (prevalence about 100/100 000) and three (V, VI and VII) are from an area of average risk in Finland (prevalence 50-60/100 000). ${ }^{22}$ In family I there were seven siblings, one with CDMS and one with CPMS. In family II there were six siblings, one with CDMS and one with CPMS. In family III there were three siblings, two with CDMS, one with CPMS, and the father had had optic neuritis. In family IV there were ten siblings, four with CDMS. In families V and VI there were five siblings, two with CDMS. In families VII and VIII there were two and four siblings, respectively, one of them and the mother had CDMS. In family IX there were six siblings, one of them, an aunt and her son (cousin) had CDMS.

All subjects were interviewed and clinically examined by a neurologist (JW), and their clinical records were obtained from health centres and hospitals. Special attention was paid to risk factors for stroke such as cardiac disease, hypertension and smoking. Blood glucose and cholesterol levels were not studied. The disability of MS patients was evaluated using Kurtzke's expanded disability status scale. ${ }^{23}$ Age of onset was defined as the first episode of neurological dysfuncton suggesting demyelinating disease.

A $1.0 \mathrm{~T}$ superconductive magnet (Siemens 
Table 1 Criteria for lesions found on MRI considered suggestive of MS

Criteria $\mathrm{I}^{24}$ : Applied to subjects under age 50 without risk factors for stroke.

A) Four lesions present.

B) Three lesions present, one periventricular.

Lesion diameter in both cases greater than $3 \mathrm{~mm}$.

Criteria $\mathrm{II}^{25}$ : Applied to subjects over age 50, and to those possessing risk factors for stroke.

A) Lesion size at least $6 \mathrm{~mm}$

B) Infratentorial location.

B) Infratentorial location.

Two out of three features $(A, B, C)$ required.
Magnetom) was used for cranial MRI studies. The spinal cord was not examined. Spin echo axial, coronal and sagittal images were obtained with sequencies $T_{2} R_{250 / 22}$ and $\mathrm{TR}_{2500 / 90}$. All slices were $5 \mathrm{~mm}$ thick, with a $1 \mathrm{~mm}$ interslice gap and a field of view of $23 \mathrm{~cm}$. An image matrix of $256 \times 256$ was used with one excitation. Imaging required 10.44 minutes. The scans were analysed by certified neuroradiologists (OS, LV) blinded to the clinical diagnosis. The number, size and location of white matter lesions for all scans were recorded. Two types of criteria for MRI findings were applied to improve their specifcity for demyelination (table 1 ).

Patients with CDMS or CPMS were informed of the MRI findings but not those with normal history and examination. No further examinations will be performed unless they are justified by new symptoms or signs.

\section{Results}

Forty four subjects, 17 patients with MS and 27 asymptomatic siblings were examined with MRI. Four subjects could not be examined. Of these, three were asymptomatic; two of them had claustrophobia and one had a cardiac pacemaker. The fourth, with CDMS (IV/1), was too disabled to be examined.

A grossly abnormal scan of a patient (III/2) with CPMS who was asymptomatic at the time of examination is shown in fig 1A. A scan of a clinically healthy subject (IV/8) with multiple periventricular lesions fulfilling criteria II is shown in fig B. Lesions of an asymptomatic subject (IV/7) fulfilling criteria I but not criteria II are illustrated in fig C.

The distribution of MRI findings according to both criteria is shown in table 2 . Of the 17 subjects with MS $13(76 \%)$ fulfilled both criteria I and criteria II for specificity of the lesions. Eleven (79\%) of the 14 patients with CDMS and two $(67 \%)$ of the three patients with CPMS fulfilled both criteria. Two

Table 2 Distribution of MRI findings by different criteria among patients with multiple sclerosis (MS) and their asymptomatic siblings

\begin{tabular}{|c|c|c|c|c|}
\hline & \multirow[b]{2}{*}{$n$} & \multirow{2}{*}{$\begin{array}{l}\text { Lesions found } \\
\text { on MRI }\end{array}$} & \multicolumn{2}{|c|}{ Lesions fulfilling criteria } \\
\hline & & & $\bar{I}$ & $I I$ \\
\hline $\begin{array}{l}\text { All MS patients } \\
\text { CDMS } \\
\text { CPMS } \\
\text { Asymptomatic siblings age }<50 \text { yrs (mean 39) } \\
\text { Asymptomatic siblings age } \geqslant 50 \text { yrs (mean 57) }\end{array}$ & $\begin{array}{r}17 \\
14 \\
3 \\
16 \\
11\end{array}$ & $\begin{array}{l}15(88 \%) \\
12(86 \%) \\
3(100 \%) \\
6(38 \%) \\
8(73 \%)\end{array}$ & $\begin{aligned} 13(76 \%) \\
11(79 \%) \\
2(67 \%) \\
2(13 \%) \\
3(27 \%)\end{aligned}$ & $\begin{aligned} & 13(76 \%) \\
& 11(79 \%) \\
& 2(67 \%) \\
&-1(9 \%)\end{aligned}$ \\
\hline
\end{tabular}

CDMS = clinically definite multiple sclerosis; CPMS = clinically probable multiple sclerosis. patients, one with CDMS (VI/2) and one with CPMS (II/5) had lesions but these met neither criteria, and two patients with CDMS (III/1 and IX/2) had no lesions visible on MRI.

Fourteen $(52 \%)$ of the 27 asymptomatic siblings had lesions on MRI. Five of them, all over age 50, had risk factors for stroke. In the under 50 age group six $(38 \%)$ out of 16 asymptomatic siblings had lesions, but only two $(13 \%)$ of them (I/2 and VIII/2) had lesions fulfilling criteria I (table 2). In the over 50 age group eight $(73 \%)$ out of 11 asymptomatic siblings had lesions. The lesions of three subjects (IV/7, IV/8 and V/1) fulfilled criteria I whereas only one had lesions fulfilling also criteria II (IV/8, fig B), which suggested MS in this age group. Thus altogether three $(11 \%)$ asymptomatic siblings showed lesions suggesting MS.

\section{Discussion}

White matter lesions present on MRI are not specific for MS because they can be found in common conditions such as arterial hypertension, multi-infarct dementia, vasculitis, diabetes mellitus, and cardiac disease. Lesions are often encountered in asymptomatic individuals over age 50 even in the absence of the above mentioned risk factors ${ }^{25} 26$ and thus have to be interpreted with caution.

To improve the specificity of MRI a precise evaluation of risk factors and an analysis of lesions according to their size and location are important. Lesions due to cerebrovascular disease are usually small and located in the watershed area of the superficial middle cerebral branches, in the deep perforating long medullary vessels in the centrum semiovale, or in the basal ganglia. ${ }^{25-29}$ Lesions around the horns of lateral ventricles are sometimes found among asymptomatic elderly subjects, but lesions abutting the bodies of lateral ventricles are rare in this group. ${ }^{25} \mathrm{MS}$ lesions tend to be larger and are usually located around lateral ventricles as well as around the third and fourth ventricles. ${ }^{16252729-31}$ Lesions around the bodies of lateral ventricles are regarded rather specific for MS, whereas such lesions are rarely seen in asymptomatic individuals. ${ }^{25}$ Infratentorial lesions also suggest demyelination. ${ }^{2527}$ MS lesions tend to be more focal than in the deep white matter infarction, in which lesions are often ill defined and more confluent. ${ }^{32}$ Nevertheless, no lesion can be regarded as pathognomic of MS, stressing the fact that the diagnosis is still primarily clinical.

In our series 14 (52\%) out of 27 asymptomatic siblings showed lesions on MRI. Eight (73\%) of the 11 asymptomatic siblings over age 50 had these lesions. The high frequency of the lesions among asymptomatic subjects is evidence of the sensitivity of MRI. To increase the specificty, we analysed the lesions according to their size and location using two criteria for findings suggesting MS. The prevalence of non-demyelinative white matter lesions increases with advancing age, ${ }^{2633}$ therefore we used the stricter criteria II for subjects over age 50 . When Fazekas $e t a^{25}$ used criteria II none of 
Figure (A) MRI of a 39 year old woman (III/2) with clinically probable MS. She had had episodes of paresthesiae, positive Lhermitte's sign, and pathological IgG-index of the CSF. At the time of examination she was asymptomatic. $A$ large asymptomatic. A large
high signal area can be seen in the left centrum semiovale, a confluent mass behind the right lateral ventricle, and small lesions bilaterally around lateral ventricles. Six months after the examination she experienced right hemiparesis and paresthesias, and her diagnosis was confirmed as CDMS; (B) MRI of a 54 year old woman (IV/8) who had had urge incontinence of micturition for 30 years but no other symptoms or signs suggestive of MS. She did not have any risk factors for stroke. Multiple plaques can be seen around the lateral ventricles, subcortically, and in the centrum semiovale bilaterally; (C) MRI of an asymptomatic 56 year old man (IV/7) with arterial hyperteission. Multiple white matter lesions are seen in the centrum semiovale and subcortically in both hemispheres.

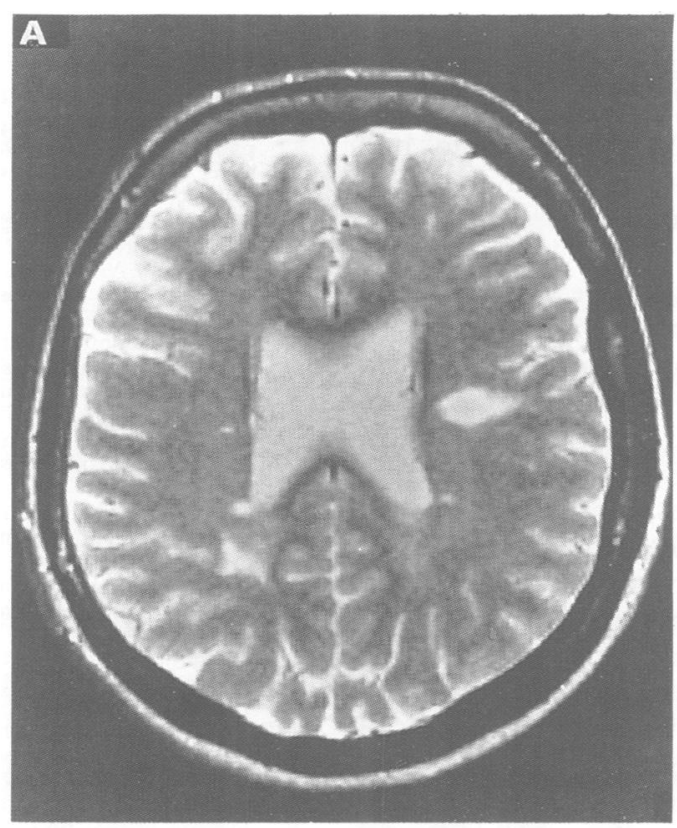

the 41 asymptomatic subjects over age 50 showed lesions suggesting demyelination but $88 \%$ of MS patients had these lesions. In the series of Yetkin $e t a^{34}$ only one of the control group consisting of 100 healthy volunteers, 60 subjects with hypertension and eight patients with dementia showed lesions meeting criteria II. Thus very high specificity for MS can be obtained with these criteria, and they would then be preferred in the scans of elderly subjects. However, the use of such stringent criteria may exclude some cases of MS, and the relaxation of these criteria would inevitably introduce several subjects with vascular disease. Consequently, it seems that the most reliable interpretations can be drawn from the studies of subjects in age group under 50 or even preferably in age group under 40 , in which the prevalence of white matter lesions in the general population is extremely low. When studying families multiply affected by MS it is difficult to restrict MRI studies only to sibships under age 40 , because of the late diagnosis of MS in many cases. Given the limitation that criteria II may exclude cases of MS the very low frequency of false positives encountered with these criteria justifies their use in order to diminish the most probable bias in these studies: classifying lesions due to vascular disease as demyelinating.

Thirteen (76\%) of 17 patients with CDMS or CPMS fulfilled both criteria for specificity of the lesions. Hence, the sensitivity was not reduced when criteria II were applied. Family IV represents one of the largest reported aggregation of siblings with CDMS: four out of ten siblings had CDMS. In this family there was one possible subclinical cases of MS, too. As has been shown earlier ${ }^{35}$ multiple lesions can be found on MRI despite normal clinical findings among MS patients, most probably because up to $75 \%$ of lesions identified by MRI can be clinically "silent". ${ }^{36}$ Even such large and numerous lesions that were found in fig A did not cause any symptoms or signs at the time of examination.

Among clinically asymptomatic siblings under age 50 none had lesions fulfulling criteria II. Two subjects (I/2 and VIII/2) showed lesions fulfilling criteria $\mathrm{I}$, which in this age group suggests demyelination. In the age group over 50 there were two asymptomatic men (IV/6 and V/1, both possessing risk factors for stroke) whose lesions fulfilled criteria I, but not criteria II. These are more likely to be nondemyelinating lesions rather than evidence for subclinical MS. Thus three (11\%) out of 27 asymptomatic individuals, two under age 50 fulfilling criteria I and one over age 50 fulfilling criteria II, were candidates for having subclinical MS. All these subjects were from multiplex families from the area of very high risk for MS and therefore the results can not be extrapolated to families with a single MS case. Follow up of these subjects for future symptoms or new lesions by repeated MRI will be of interest. In the series of Lynch et $a l^{19}$ the lesions of two (9.5\%) out of 21 asymptomatic subjects over age 50 in MS families fulfilled criteria II. They may represent subclinical 
demyelination in the age group over 50 , a finding quite similar to ours $(1 / 11,9 \%)$. In the age group under 50 Lynch et al found four asymptomatic subjects out of 24 who had lesions, but none of these seemed to meet the criteria I used in our study.

Genetic susceptibility to MS has become a field of intensive research. ${ }^{5}$ The occurrence of subclinical disease among asymptomatic siblings of MS patients has a strong impact on genetic investigation. It is an important confounding factor in family studies resulting in false phenotypes. Using age of onset correction $^{37}$ in linkage analyses because of reduced age-dependent penetrance decreases the effect of misdiagnosis in subjects under the age of 60 , by which time the maximum penetrance of MS has been achieved. ${ }^{21}$ There may, however, be subjects whose disease never becomes manifest. This may either be a consequence of variation of environmental or genetic factors, ${ }^{11}$ or represent an intermediate on a continuum leading eventually to $M S^{12}$ Of the genetic linkage analysis sibpair analysis is not sensitive to the effects of reduced penetrance or subclinical disease since only the affected subjects are studied but a lot of genetic information is lost compared with classical linkage analysis. ${ }^{37}$ Clinical follow up $^{38}$ and MRI studies of the first-degree relatives of MS patients when using them as healthy controls in family studies would therefore greatly aid the efforts to identify the susceptibility genes and the environmental factors leading to the disease.

This study was supported by grants from Sigrid Juselius foundation, Paulo foundation, Finnish Neurology Foundation, and the Research Foundation of Orion.

1 Pratt RTC, Compston AD, McAlpine D. The familial incidence of disseminated sclerosis and its significance. Brain 1951;74:191-232.

2 Miller R. Genetic aspects of multiple sclerosis. Arch Neurol Psychiat (Chicago) 1953;70:733-40

3 Shapira K, Poskanzer DC, Miller H. Familial and conjugal multiple sclerosis. Brain 1963;86:315-32.

4 Mackay RP, Myrianthopoulos NC. Multiple sclerosis in twins and their relatives. Arch Neurol 1966;15:449-62.

5 Compston A. Genetic factors in the aetiology of multiple sclerosis. In: McDonald WI, Silberberg DH, eds. Multiple sclerosis. Butterworths, London 1986:56-73.

6 Sadovnik AD, Baird PA, Ward RH. Multiple sclerosis: updated risks for relatives. Am $\mathcal{f}$ Med Genet 1988; 29:533-41.

7 Wikström J, Kinnunen E, Porras J. The age specific prevalence ratio of familial mulstiple sclerosis. Neuroepidemiology 1984;3:74-81.

8 Uitdehaag BMJ, Polman $\mathrm{CH}$, Valk J, et al. Magnetic resonance imaging studies in multiple sclerosis twins. $\mathcal{f}$ Neurol Neurosurg Psychiatry 1989;52:1417-9.

9 Williams A, Eldridge R, McFarland H, et al. Multiple sclerosis in twins. Neurology 1980;30:1139-47.

$10 \mathrm{McFarland}$ HF, Patronas NJ, McFarlin DE, et al. Studies of multiple sclerosis in twins using MRI (abstract). Neurology 1985;35(suppl 1):137.
11 Duquette P, Charest L. Cerebrospinal fluid findings in healthy siblings of multiple sclerosis patients. Neurology 1986;36:727-29.

12 Nuwer MR, Visscher BR, Packwood JW, Namerow WS. Evoked potential testing in relatives of multiple sclerosis patients. Ann Neurol 1985;18:30-4.

13 Gilbert JJ, Sadler M. Unsuspected multiple sclerosis. Arch Neurol 1983;40:533-6.

14 Phadke JG, Best PV. Atypical and clinically silent multiple sclerosis: a report of 12 cases discovered unexpectedly at necropsy. $¥$ Neurol Neurosurg Psychiatry 1983;46:414-20. necropsy. 7 Neurol Neurosurg Psychiatry 1983;46:414-20.
McDonald WI. Diagnosis of multiple sclerosis. BMF 1989;299:635-7.

16 Cutler J, Aminoff MJ, Brant-Zawadski M. Evaluation of patients with multiple sclerosis by evoked potentials and MRI: a comparative study. Ann Neurol 1986;20:645-8.

17 Giesser BS, Kurtzberg D, Vaughan HG, et al. Trimodal evoked potentials compared with magnetic resonance imaging in the diagnosis of multiple sclerosis. Arch Neurol 1987;44:281-4.

18 Sola P, Scarpa M, Faglioni P, et al. Diagnostic investigations in MS: which is most sensitive? Acta Neurol Scand 1989;80:394-9.

19 Lynch SG, Rose JW, Smoker W, et al. MRI in familial multiple sclerosis. Neurology 1990;40:900-3.

20 Kinnunen E, Wikström J, Porras J, Palo J. The epidemiology of multiple sclerosis in Finland: Increase of prevalence and stability of foci in high-risk areas. Acta Neurol Scand and stability of foci

21 Poser CM, Paty DW, Scheinberg L, et al. New diagnostic criteria for multiple sclerosis: guidelines for research protocols. Ann Neurol 1983;13:263-5.

22 Wikström J, Palo J. Studies on the clustering of multiple sclerosis in Finland I: comparison between the domiciles and places of birth in selected subpopulations. Acta Neurol Scand 1975;51:85-9.

23 Kurtzke JF. Rating neurological impairment in multiple sclerosis: an expanded disability status scale (EDSS). Neurology 1983;33:144-52.

24 Paty DW, Oger JJF, Kastrukoff LF, et al. MRI in the diagnosis of MS: A prospective study with comparison of clinical evaluation, evoked potentials, oligoclonal banding, and CT. Neurology 1988;38:180-5.

25 Fazekas F, Offenbauer H, Fuchs S, et al. Criteria for an increased specificity of MRI interpretations in elderly subjects with suspected multiple sclerosis. Neurology subjects with susp

26 Hunt AL, Orrison WW, Yeo RA, et al. Clinical significance of MRI white matter lesions in the elderly. Neurology 1989;39:1470-4.

27 Uhlenbrock D, Sehlen S. The value of T1-weighted images in the differentiation between MS, white matter lesions, and subcortical arteriosclerotic encephalopathy (SAE). Neuroradiology 1989;31:202-12.

28 Lechner H, Schmidt R, Götz B, et al. Nuclear magnetic resonance image white matter lesions and risk factors for stroke in normal individuals. Stroke 1988;19:263-5.

29 Ormerod IEC, Roberts RC, du Boulay EPGH, et al. NMR in multiple sclerosis and cerebral vascular disease. Lancet 1984;2:1334-5.

30 Gebarski SS, Gabrielsen TO, Gilman S, et al. The initial diagnosis of multiple sclerosis: clinical impact of magnetic diagnosis of multiple sclerosis: clinical impact of magn

31Sheldon J, Siddharthan R, Tobias J, et al. MR imaging of multiple sclerosis: comparison with clinical and CT examinations in 74 patients. AfNR 1985;6:683-90.

32 Bradley WG. White matter disease in the elderly. In: Kressel HY, Modic M, Murphy W, eds. Syllabus: special course MR 1990. RSNA publications, Oak Brook 1990:103-7.

33 Hendrie HC, Farlow MR, Austrom MG. Foci of increased signal intensity on brain MR scans of healthy elderly subjects. AFNR 1989;10:703-7.

34 Yetkin FZ, Haughton VM, Papkie RA, et al. Multiple sclerosis: specificity of MR for diagnosis. Radiology 1991;178:447-51.

35 Palo J, Ketonen L, Wikström J. A follow-up study of very low field MRI findings and clinical course in multiple sclerosis. F Neurol Sci 1988;84:177-87.

36 Jacobs L, Kinkel WR, Polachini I, et al. Correlations of nuclear magnetic resonance imaging, computerized tomography, and clinical profiles in multiple sclerosis. tomography, and clinical

37 Ott J. Haplotype sharing in affected sibs. Analysis of human genetic linkage. Baltimore \& London: The Johns Hopkins Univ Press, 1985:164-5.

38 Goodkin DE, Doolittle TH, Hauser SS, et al. Diagnostic criteria for multiple sclerosis research involving multiply affected families. Arch Neurol 1991;48:805-7. 